\title{
Direct Probing of Gap States and Their Passivation in Halide Perovskites by High-Sensitivity, Variable Energy Ultraviolet Photoelectron Spectroscopy
}

\author{
Published as part of The Journal of Physical Chemistry virtual special issue "D. D. Sarma Festschrift". \\ Igal Levine, ${ }^{*}$ \& Kohei Shimizu, ${ }^{\&}$ Alberto Lomuscio, Michael Kulbak, Carolin Rehermann, Arava Zohar, \\ Mojtaba Abdi-Jalebi, Baodan Zhao, Susanne Siebentritt, Fengshuo Zu, Norbert Koch, Antoine Kahn, \\ Gary Hodes, Richard H. Friend, Hisao Ishii,* and David Cahen
}

Cite This: J. Phys. Chem. C 2021, 125, 5217-5225

Read Online

ACCESS | Llll Metrics \& More | 国 Article Recommendations ｜ｏ） Supporting Information

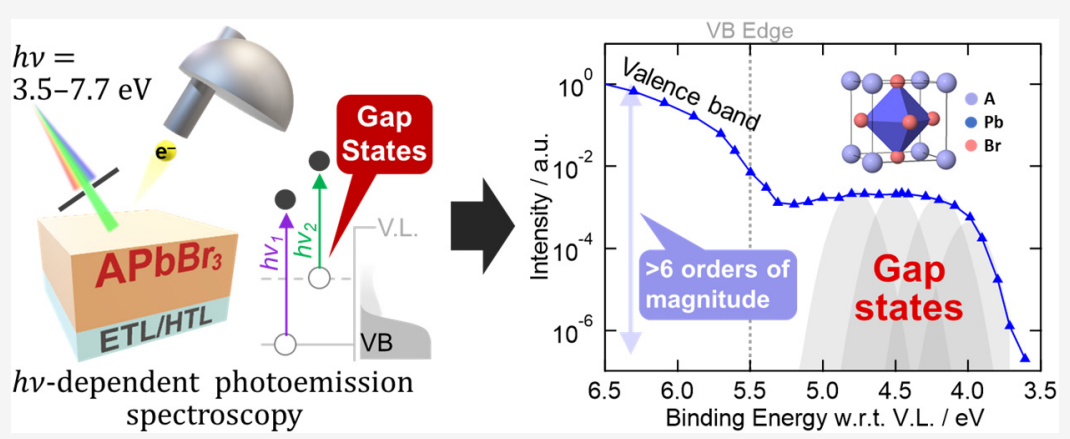

ABSTRACT: Direct detection of intrinsic defects in halide perovskites (HaPs) by standard methods utilizing optical excitation is quite challenging, due to the low density of defects in most samples of this family of materials $\left(\leq 10^{15} \mathrm{~cm}^{-3}\right.$ in polycrystalline thin films and $\leq 10^{11} \mathrm{~cm}^{-3}$ in single crystals, except melt-grown ones). While several electrical methods can detect defect densities $<10^{15}$ $\mathrm{cm}^{-3}$, such as deep level transient spectroscopy (DLTS) or thermally stimulated current (TSC), they require preparation of ohmic and/or rectifying electrical contacts to the sample, which not only poses a challenge by itself in the case of HaPs but also may create defects at the contact-HaP interface and introduce extrinsic defects into the HaP. Here, we show that low-energy photoelectron spectroscopy measurements can be used to obtain directly the energy position of gap states in $\mathrm{Br}$-based wide-bandgap ( $\mathrm{E}_{\mathrm{g}}>2 \mathrm{eV}$ ) HaPs. By measuring HaP layers on both hole- and electron-contact layers, as well as single crystals without contacts, we conclude that the observed deep defects are intrinsic to the $\mathrm{Br}$-based $\mathrm{HaP}$, and we propose a passivation route via the incorporation of a $2 \mathrm{D}$ forming ligand into the precursor solution.

\section{INTRODUCTION}

The maximum theoretical efficiency of a solar cell is limited by thermodynamics, as presented by Shockley and Queisser (SQ) in 1961. ${ }^{1}$ Apart from the thermodynamic limitation, structural defects (such as point defects, grain boundaries, dislocations, interfacial defects, etc.) can cause a reduction in device efficiency, mainly due to voltage loss via nonradiative recombination of photoexcited charge carriers. Voltage loss can also occur, even in an ideal absorber, by other cell components (e.g., energy mismatch between the absorber and (selective) contacts). Nonradiative recombination of electronic charge carriers in a semiconductor is usually caused by gap states that lie deep in the bandgap $\left(E_{\mathrm{g}}\right)$ and serve as recombination centers. Therefore, detecting and characterizing deep defect states in the bandgap of semiconductors are important for identifying limiting factors in electronic devices and possible defect-related degradation mechanisms, ${ }^{2}$ and they constitute a necessary step for further device development as well as often presenting a technical and scientific challenge.

While there exists a variety of sensitive methods for detecting gap states in semiconductors, the "ideal" method for detecting them, determining their energy position within the bandgap, and assessing their quantity would be one that fulfills the following two conditions: (1) it does not require additional preparation steps that might alter the sample (e.g.,

Received: December 31, 2020

Revised: February 8, 2021

Published: March 2, 2021 
deposition of electrical contacts); (2) the analysis of the experimental raw data will require the least postprocessing steps and be based on the most straightforward model, i.e., with as few empirical assumptions as possible. In principle, such a direct experimental method is ultraviolet photoemission spectroscopy (UPS). However, in a conventional UPS setup employing a helium discharge lamp, the signal-to-noise ratio (2-3 orders of magnitude) limits the density of gap states that can be detected. This limitation is especially pronounced in the case of halide perovskites ( $\mathrm{HaPs}$ ), where the density of defects was found to be $10^{15} \mathrm{~cm}^{-3}$ and below. ${ }^{3-5}$ Until now three methods have shown promise to overcome this problem: remove carefully all parasitic components due to satellite emissions from the helium discharge lamp, from experimental (He I, II) UPS spectra, ${ }^{6}$ use laser-based UV sources with lower energies (than $\mathrm{He} \mathrm{I}$ ), to significantly enhance the ionization cross-section for photoemission from trap states, ${ }^{7}$ and use modulated surface photovoltage (SPV) for detecting band-totrap or trap-to-band optical transitions. ${ }^{4}$

Several efforts have been made to improve the detection limits of UPS: ${ }^{8,9}$ Sueyoshi et al. ${ }^{8}$ achieved a detection limit using UPS of better than $10^{18}$ states $\mathrm{cm}^{-3}$ with a He I light source, dispersed with a grating, two-dimensional photoelectron detection, and the suppression of electrical noise from connectors and cables. This resulted in "high-sensitivity UPS (HS-UPS)" and made UPS analysis using the logarithm of the detector signal a viable option. ${ }^{10-12}$ Boehm et al. showed that by using the narrow line width $10.2 \mathrm{eV}$ hydrogen Lyman- $\alpha$ emission as the source instead of the He I light source, the $S / N$ ratio can be improved $\sim 100$-fold, while also minimizing radiation-induced damage to the probed sample. ${ }^{13}$ It has been shown recently that photoemission spectroscopy, using a light source with photon energies, $h \nu$, tunable and smaller than that of He I, namely $h \nu$-dependent HS-UPS, provides even higher sensitivity and a wider dynamic range, of more than 6 orders of magnitude, than regular UPS. Because of such high-sensitivity, HS-UPS can yield valuable data regarding the energy position of the gap states and their density. ${ }^{14,15}$ Furthermore, the $h \nu$ dependence of the photoemission intensity from the occupied states can help to approximate the wave function of the initial state because the photoemission intensity from a constant initial energy state can be related to the Fourier transform of the initial states. ${ }^{16}$

Using different methods such as modulated $\mathrm{SPV}^{4}$ and photo-Hall measurements, ${ }^{17}$ it has been shown that Br-based $\mathrm{HaPs}\left(E_{\mathrm{g}} \sim 2.3 \mathrm{eV}\right)$, contain several distributions of gap states. Incorporation of a $2 \mathrm{D}$-forming ligand into $3 \mathrm{D} \mathrm{HaPs}$ thin films has shown great potential for improving the stability and free carrier lifetime of the resulting mixed 2D/3D film. ${ }^{18}$ Recently both Cheng et al. ${ }^{19}$ and Zhao et al. ${ }^{20}$ found that the addition of phenylethylammonium bromide (PEABr) to the pure $3 \mathrm{D}$ inorganic $\mathrm{CsPbBr}_{3} \mathrm{HaP}$ precursor solution enhances the photoluminescence quantum efficiency (PLQE) of the resulting $2 \mathrm{D} / 3 \mathrm{D}$ film. Zhao reported an increase from $\sim 1 \%$ to $12 \%$ in the PLQE, and when an additional additive, tetraphenylphosphonium chloride (TPPCl), was added, the PLQE was further increased to $61 \% .^{20}$ However, a direct link between the distribution of gap states and the PLQE was not established.

In this work, we employ the HS-UPS method to determine both the energy position and density of gap states in several Brbased lead HaPs. To distinguish between tail, shallow, and deep gap states, due to the relatively wide bandgap of the HaPs studied here $(\sim 2.3 \mathrm{eV})$, we denote gap states as deep if they are $>300 \mathrm{meV}$ from the band edges, shallow if they are located between 150-300 meV from the band edges, and tail states (i.e., gap states that do not act as recombination centers or as trap states at room temperature (RT)) if they are connected to the extended band edges and positioned less than $150 \mathrm{meV}$ from the band edges. ${ }^{2}$

To set the stage, we present first the results from purely optical methods-photothermal deflection spectroscopy (PDS) and photoluminescence (PL). Results from these measurements do not indicate the presence of deep gap states. Next, the results of HS-UPS measurements are shown, and they indeed enable direct detection of deep defects in the pure 3D $\mathrm{Br}-\mathrm{HaP}$ thin films as well as in single crystals. In contrast, for a passivated mixed $2 \mathrm{D} / 3 \mathrm{D} \mathrm{HaP}$, only a very low concentration of defects, if indeed any, is detected.

\section{METHODS}

HS-UPS was conducted with a home-built apparatus. ${ }^{8,15,22}$ Two different white light sources, a $150 \mathrm{~W} \mathrm{D}_{2}$ lamp (Hamamatsu Photonics L1835) and a $500 \mathrm{~W}$ Xe lamp (Ushio Inc. UXL-500D), were installed and one or the other could be selected by changing a reflecting mirror. The light was monochromatized by a zero-dispersion double monochromator (BUNKOUKEIKI M25GTM-DZ) to eliminate stray light. The relative intensity of stray light was less than $1 \times 10^{-9}$ at a wavelength of $632.8 \mathrm{~nm}$. Second-order light was also suppressed using band-pass filters. The incidence angle was 55 deg from the surface normal, and photoelectrons were collected at the normal emission angle with a $120 \mathrm{~mm}$ hemispherical analyzer (PSP RESOLVE 120). The overall energy resolution was about $200 \mathrm{meV}$, which was determined by fitting the Fermi edge of a gold film with Fermi-Dirac and Gaussian distributions. To normalize the photoemission intensity, the incident, monochromatized photon flux was recorded by a photomultiplier tube (Hamamatsu Photonics $\mathrm{R} 376$ ); therefore, the signal intensity is given in arbitrary units (a.u.).

Samples were removed from their inert atmosphere container and introduced to the preparation chamber within $10 \mathrm{~min}$, as received, except for the single crystal of $\mathrm{MAPbBr}_{3}$, which was first cleaved (in ambient) and the cleavage plane was polished with DMF in ambient just before its introduction into the chamber. The time that elapsed between the final polish and closing the chamber was about $5 \mathrm{~min}$. After the preparation chamber was pumped for $45 \mathrm{~min}$, the sample was transferred to the analysis chamber whose base pressure was $<2 \times 10^{-8} \mathrm{~Pa}$.

The $\left(\mathrm{FA}_{0.85} \mathrm{MA}_{0.1} \mathrm{Cs}_{0.05}\right) \mathrm{PbBr}_{3}$ (hereinafter termed "Mix$\mathrm{APbBr}_{3}$ ") thin films were prepared using the procedure that is described in detail in ref 4 . The precursor salts were $\mathrm{FABr}$ (Dyesol, >99\%), MABr (Dyenamo, 99.99\%), CsBr (abcr, 99.999\%), and $\mathrm{PbBr}_{2}$ (TCI, > 98\%). The thickness of the MixAPbBr $r_{3}$ films was about $300 \mathrm{~nm}$. $\mathrm{MAPbBr}_{3}$ single crystals were prepared from $\mathrm{MABr}$ (Dyesol, > 99.99\%) and $\mathrm{PbBr}_{2}$ (Dyesol) precursors using the antisolvent method as described detail in ref 23 . The mixed $2 \mathrm{D} / 3 \mathrm{D}$ films were prepared using $\mathrm{PEABr}$ with the standard precursors of $\mathrm{CsPbBr}_{3} 3 \mathrm{D}$ perovskites. The molar ratio of the perovskite precursors with lead bromide $\left(\mathrm{PbBr}_{2}\right.$, Sigma-Aldrich, 99.999\%), cesium bromide (CsBr, Sigma-Aldrich, 99.99\%), and PEABr (Dyesol, > 99\%) in the precursor solution in DMSO was 5:5:2. In addition, 5 mol \% of TPPCl (Sigma-Aldrich, 98\%) was added to the 

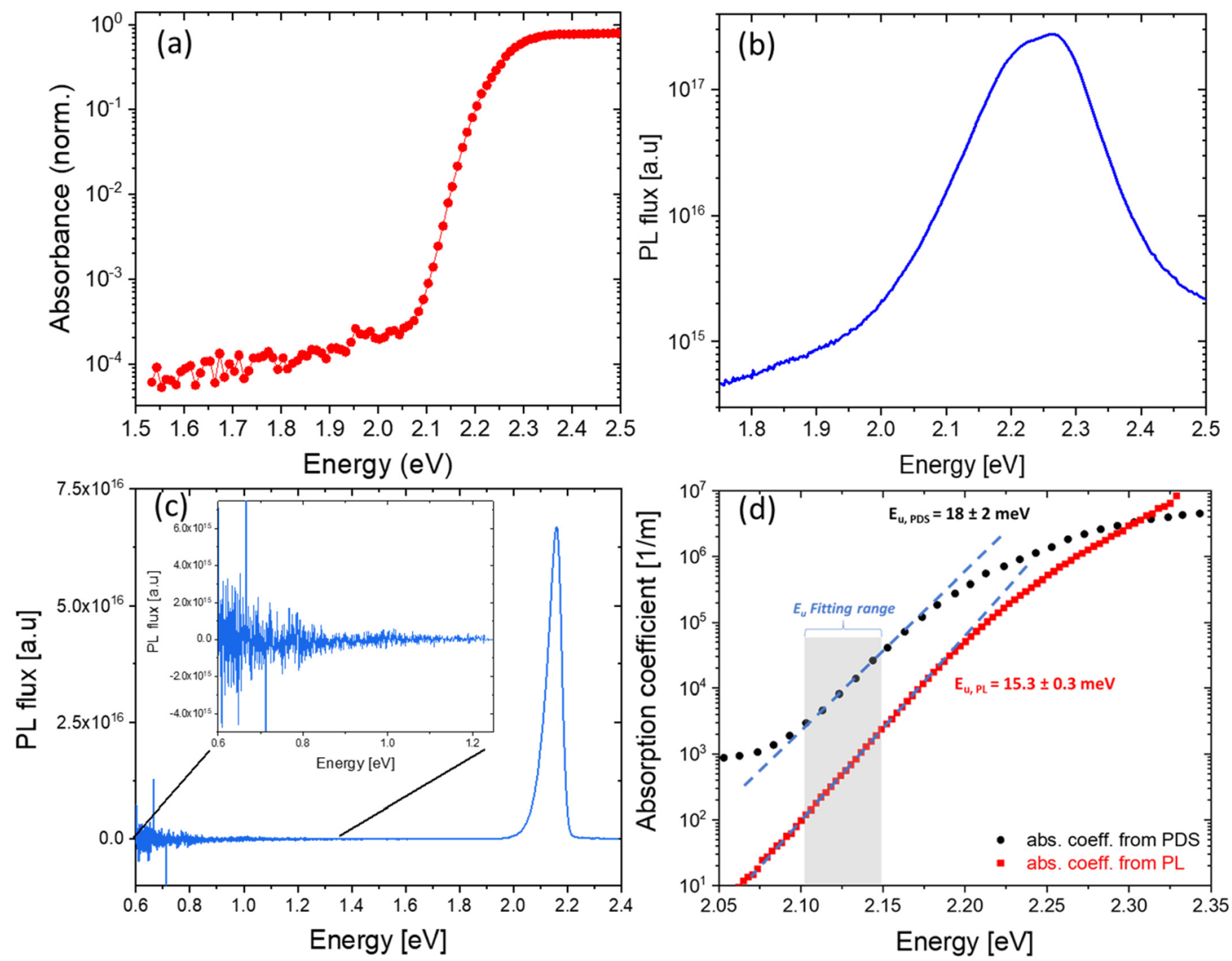

Figure 1. (a) PDS spectrum at RT of a MixAPbBr ${ }_{3}$ film deposited on quartz. (b) PL emission spectrum, recorded at RT under ambient conditions, of $\mathrm{MixAPbBr}_{3}$ deposited on quartz. (c) PL emission spectrum recorded at $10 \mathrm{~K}$ in ultrahigh vacuum (UHV) of MixAPbBr $\mathrm{Pb}_{3}$ deposited on quartz, including a close up in the region of $0.6-1.25 \mathrm{eV}$. (d) Extraction of the Urbach tail energies from the PDS and PL spectra at RT. In all cases the $x$ axes are photon energy (excitation for PDS, emission for PL).

precursor solution. The thickness of the mixed $2 \mathrm{D} / 3 \mathrm{D}$ films was about $50 \mathrm{~nm}$; further details are given in ref 20 .

The PL measurements were carried out in a home-built system with a $404 \mathrm{~nm}$ wavelength diode laser as excitation source. The samples were exposed to ambient conditions for a brief time during loading into the measurement chamber. For low temperature $\mathrm{PL}$, the samples were introduced into a helium flow cryostat. The emitted luminescence was collected by two off-axis parabolic mirrors, focused into a fiber, spectrally resolved by a monochromator, and detected by Si-CCD and InGaAs arrays. The raw data are corrected for the spectral response of the system using a commercial calibration lamp with a known spectrum.

PDS measurements were performed using monochromatic pump light ( $\mathrm{HaP}$ films on quartz substrates), which upon absorption produces a thermal gradient near the sample surface via nonradiative relaxation-induced heating. This results in a refractive index gradient in the area surrounding the sample surface. This refractive index gradient is further enhanced by immersing the sample in an inert liquid FC-72 Fluorinert (3 M Company) which has a high refractive index change with temperature. A fixed wavelength $\mathrm{CW}$ laser probe beam is passed through this refractive index gradient producing a deflection proportional to the absorbed light at that particular wavelength, which is detected by a photodiode and lock-in amplifier combination. Scanning through different wavelengths yields the complete absorption spectrum. The sample was very briefly exposed to ambient conditions during loading and then immersed in an inert liquid during the measurement at room temperature.

\section{RESULTS AND DISCUSSION}

One of the most sensitive optical absorption methods is PDS. $^{24}$ Figure 1a shows the PDS spectrum of the mixed MixAPbBr 3 , with very low sub-bandgap absorption. It indicates that if there are any shallow or deep defects in $\mathrm{MixAPbBr}_{3}$ film, their densities approach the PDS detection limit and cannot be assessed in a reliable manner using PDS. Figure $1 \mathrm{~b}$ shows the $\mathrm{PL}$ spectrum of the $\mathrm{MixAPbBr}_{3}$ film at RT, exhibiting a slightly asymmetric peak shape and a peak position at $2.27 \mathrm{eV}$, in agreement with the literature bandgap. ${ }^{25}$ It has been shown that low temperature PL measurements can be used as a sensitive method to probe sub-bandgap defect states in MAPI. ${ }^{26}$ To investigate if defect-related PL emission bands are present in $\mathrm{MixAPbBr}_{3}$, a PL spectrum was taken at $T=10$ $\mathrm{K}$ (Figure 1c) and in accordance with the literature, ${ }^{27}$ the near bandgap PL emission energy is shifted to a lower value of 2.16 $\mathrm{eV}$ (possibly due to primary emission from bound excitons), while its asymmetric shape suggests a contribution of tail states and shallow defects to the PL signal down to roughly $1.9 \mathrm{eV}$ (260 meV below the $10 \mathrm{~K}$ bandgap). However, besides the decreased $S / N$ ratio at lower PL emission energies, no deep defect-related transitions are observed over the range investigated down to $0.6 \mathrm{eV}$, in agreement with the PDS data (the artificial increase in the noise level at low energies is due to the different detectors used for each energy range). With respect to tail states, the Urbach energy that is extracted 

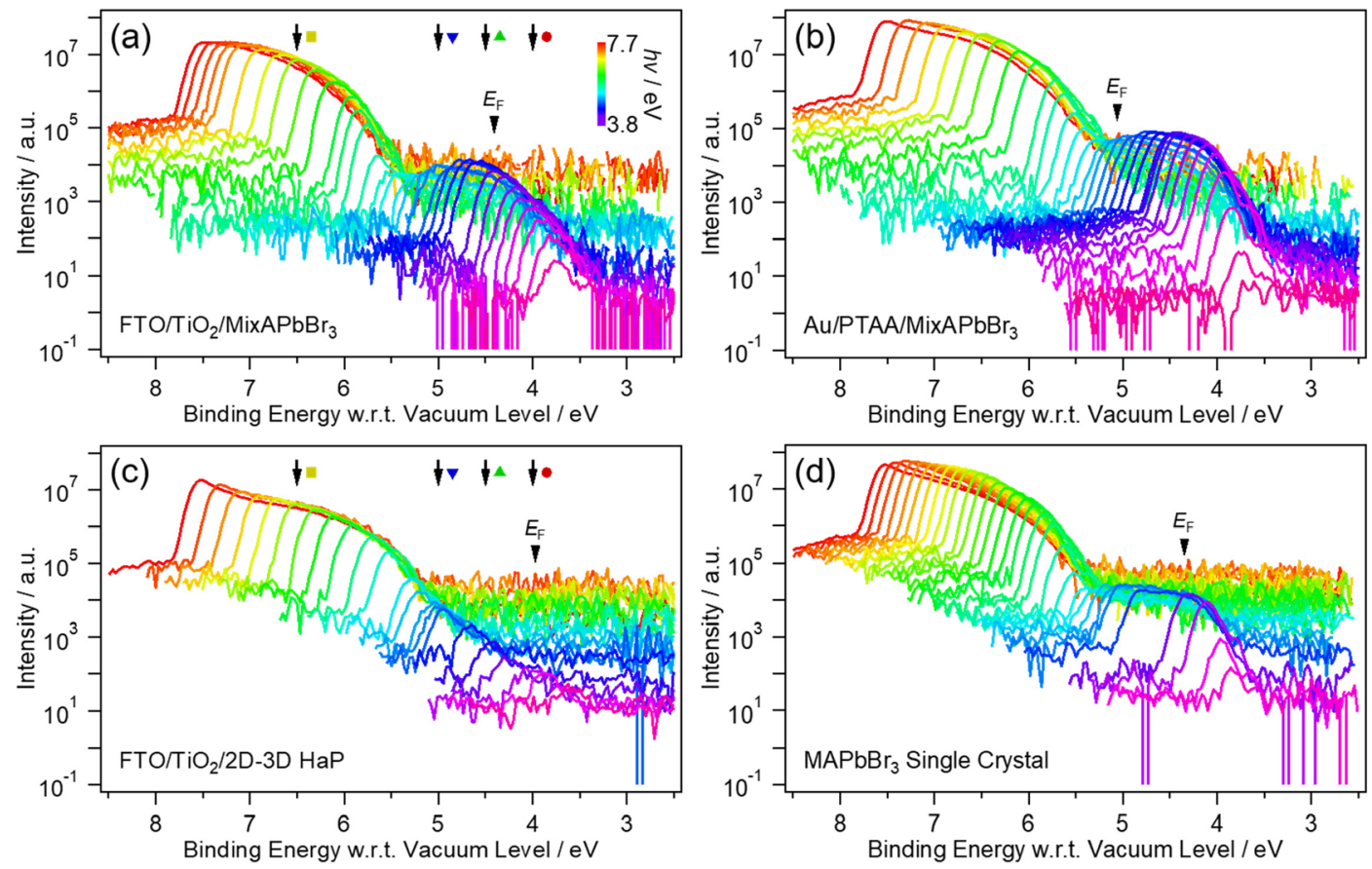

Figure 2. Variable energy HS-UPS measurements of (a) MixAPbBr $r_{3}$ deposited on glass/Au/PTAA substrate. (b) $\mathrm{MixAPbBr}_{3}$ deposited on glass/ FTO $/ \mathrm{TiO}_{2} / \mathrm{mp}-\mathrm{TiO}_{2}$ substrate. (c) $2 \mathrm{D} / 3 \mathrm{D} \mathrm{Br}$-based $\mathrm{HaP}$ deposited on glass $/ \mathrm{FTO} / \mathrm{TiO}_{2} / \mathrm{mp}-\mathrm{TiO}_{2}$. (d) $\mathrm{MAPbBr}_{3}$ single crystal. Spectra are colored according to used photon energy ranging from $7.7 \mathrm{eV}$ (red) to $3.8 \mathrm{eV}$ (purple). Triangles accompanied by the text " $E_{\mathrm{F}}$ " indicate the Fermi level, and arrows are the energies featured in Figure 4. All $x$-axes are binding energies w.r.t. the $\mathrm{VL}$, i.e., $E_{\mathrm{B}}^{\mathrm{VL}}$.

from the PDS and PL data amounts to $18 \pm 2 \mathrm{meV}$ and $15.3 \pm$ $0.3 \mathrm{meV}$ (Figure 1d), respectively, in agreement with our previously reported value of $16 \mathrm{mV}$ from SPV measurements. ${ }^{4}$ The discrepancy between the two Urbach energies extracted here can be rationalized by the different sensitivity of the two methods: in fact, it has been shown in the past that photoluminescence spectroscopy is a powerful technique that allows absorption coefficient measurements down to values lower than what can be achieved by means of spectrophotometry and even PDS. ${ }^{28}$ This translates in a higher accuracy of the Urbach energy determined by PL. ${ }^{29}$ Additionally, the observed differences might stem from the fact that the two samples were prepared in two different laboratories (the sample for the PDS was prepared at the Weizmann Institute (WIS), while the sample for the PL was prepared at the Helmholtz Center in Berlin (HZB)).

As no clear indication for deep gap states was observed in the optical measurements, next, we turned to HS-UPS because of its very high sensitivity. Figure 2 shows the HS-UPS spectra for several Br-based HaP samples. The excitation photon energy ranges from $7.7 \mathrm{eV}$ (red) to $3.8 \mathrm{eV}$ (purple) with $0.1 \mathrm{eV}$ or less intervals, and the spectra are normalized by the simultaneously measured incident photon flux. The $x$-axes give the electron binding energy with respect to the vacuum level (VL) and is referred to as $E_{\mathrm{B}}^{\mathrm{VL}}$.

In the case of an "ideal and defect-free" semiconductor, i.e., one that has negligible density of tail, shallow and deep gap states, one would expect a photoemission spectrum that exhibits a sharp signal increase near the valence band (VB) edge and negligible signal for photon energies below the ionization energy. Exemplarily, the HS-UPS spectrum of an $\mathrm{Au}$ metal film shows such an abrupt intensity change at the Fermi level $\left(E_{\mathrm{F}}\right)$ (see Figure $S 1$ in the Supporting Information). As can be seen from parts a and b of Figure 2, the VB feature of the $\mathrm{MixAPbBr}_{3}$ is observed for $E_{\mathrm{B}}^{\mathrm{VL}}>5.5 \mathrm{eV}$, and the $\mathrm{VB}$ edge, deduced from the semilog plots of the HS-UPS results, is at $E_{\mathrm{B}}^{\mathrm{VL}}=5.5 \pm 0.1 \mathrm{eV}$. This value agrees well with values extracted using standard He I UPS (averaged over different samples and measured in different laboratories; data not shown). Since the bandgap of the studied $\mathrm{Br} \mathrm{HaP}$ is $2.27 \mathrm{eV}$, this implies that the $\mathrm{CB}$ edge lies around $E_{\mathrm{B}}^{\mathrm{VL}}=3.2 \mathrm{eV}$. Notably, in addition to the VB feature, a second peak-shaped feature (which we term henceforward as the "low binding energy" feature), approximately 3 orders of magnitude lower in intensity than the VB $\left(10^{4}\right.$ compared to $\left.10^{7}\right)$, well above the noise level, is apparent in the BE range approximately between 3.5 and $5.2 \mathrm{eV}$. This low binding energy feature can be attributed to populated, deep gap states present in $\mathrm{MixAPbBr}_{3}$. Remarkably, by incorporating $\mathrm{PEABr}$ and $\mathrm{TPPCl}$ into the $\mathrm{Br}-\mathrm{HaP}$ layer, the low binding energy feature is significantly reduced as shown in the HS-UPS spectra in Figure 2c, which was further confirmed using SPV measurements (Figure S2 in the Supporting Information).

In order to ensure that the observed low binding energy feature does not originate from the underlying substrates $\left(\mathrm{TiO}_{2}\right.$ or poly[bis(4-phenyl)(2,4,6-trimethylphenyl)amine] (PTAA)), e.g., due to pinholes in the $\mathrm{MixAPbBr}_{3}$ films, a $\mathrm{MAPbBr}_{3}$ single crystal was measured, and the result is shown in Figure 2d. Based on purely electrical methods, it was found earlier that $\mathrm{HaP}$ single crystals contain a much lower density of deep gap states than thin films. ${ }^{30,31}$ However, the similarity of the HS-UPS spectra of the single crystal to that of the thin films suggests that the deep gap states of the single crystal and the thin film are rather similar in energy and also in terms of densities. The similar intensities of the low binding energy feature observed in single crystals and thin films will also be related to the fact that for HS-UPS samples up to $5 \mathrm{~nm}$ from the surface, in contrast to conventional UPS, where only the 
topmost $1-2 \mathrm{~nm}$ is probed, while using HS-UPS, the bulk of the samples is probed. Thus, the low binding energy feature is inherent to $\mathrm{Br}-\mathrm{HaP}$, which originates from deep gap states and does not depend on the cation composition.

In addition to the low binding energy feature, another striking observation in parts $a$ and $b$ of Figure 2 is the significant photoelectron signal that is observed above $E_{\mathrm{F}}$, especially pronounced for the $\mathrm{HaP}$ film on the p-type contact layer (PTAA, Figure 2b). For the $\mathrm{MixAPbBr}_{3}$ film on $\mathrm{TiO}_{2}$ and the $\mathrm{MAPbBr}_{3}$ single crystal, the signal is observed up to $\sim 0.5$ $\mathrm{eV}$ above $E_{\mathrm{F}}$, which could possibly be rationalized by a combination of two factors-SPV and the energy resolution of the experimental system. However, in the case of the $\mathrm{MixAPbBr}_{3}$ film on PTAA, where $E_{\mathrm{F}}$ lies at $E_{\mathrm{B}}^{\mathrm{VL}}=5.05 \pm$ $0.05 \mathrm{eV}$ and the signal is observed up to $1.5 \mathrm{eV}(!)$ above $E_{\mathrm{F}}$, further explanation is required. In principle, as noted above, aside from experimental resolution considerations, the most probable explanation for a significant photoelectron signal above $E_{\mathrm{F}}$ is an SPV effect, which would translate into a significant reduction in band bending and strong shift of the bands at the sample surface due to generation and separation of electron-hole pairs in the space charge region under the influence of the UV excitation used. Indeed, large SPV values (up to several hundreds of $\mathrm{meV}$ ) were recently observed by $\mathrm{Zu}$ et al. for a variety of perovskite films using variable intensities from He I UPS measurements. ${ }^{32}$ In order to rule out this possibility, similar measurements to those reported in ref 32 were performed on several $\mathrm{MixAPbBr}_{3}$ films deposited on different substrates, and the maximum observed SPV was roughly $200 \mathrm{meV}$ (Figure S3), a value that is much lower than the extension of the signal by $1.5 \mathrm{eV}$ above $E_{\mathrm{F}}$ in Figure $2 \mathrm{~b}$, and therefore cannot explain the photoelectron signal above $E_{\mathrm{F}}$. We further note that, due to the low photoexcitation energies used in the HS-UPS measurement, gap states could become populated by electrons photoexcited from the filled states of the valence band (some of them close to the top of the valence band). This could result in a slight "depletion" of these initial states, which, in combination with the relatively low density of gap states, results in a much smaller than expected net change in $E_{\mathrm{F}}$ (yet the gap states are still detected thanks to the high sensitivity of HS-UPS). A third possibility that could cause this unusual phenomenon relates to the photoexcitation of electrons trapped in gap states. Since the photon density of the light source was over 10 orders of magnitude lower than that for laser spectroscopy, two-photon processes, as seen in two-photon photoemission, are negligible. However, because primary and secondary electrons are generated within the sample during the UPS measurement, these can be trapped in deep gap states, and the trapped electrons can be excited and detected as a photoelectron signal above $E_{\mathrm{F}}$.

To obtain the density of states (DOS) from the series of UPS spectra presented in Figure 2 and to compare them among the samples, we conducted constant final state (CFS) analysis, ${ }^{33,34}$ which plots the emission intensity of photoelectrons with a specific kinetic energy $E_{K}$ at each photon energy $h \nu$ as a function of $E_{\mathrm{B}}^{\mathrm{VL}}=h \nu-E_{\mathrm{K}}$. In principle, the photoemission intensity $I_{\mathrm{PE}}$ can be expressed as

$$
I_{\mathrm{PE}} \propto D_{\mathrm{i}}\left(E_{\mathrm{B}}^{\mathrm{VL}}\right) D_{\mathrm{f}}\left(h \nu-E_{\mathrm{B}}^{\mathrm{VL}}\right) M_{\mathrm{fi}}
$$

where $D_{\mathrm{i}}$ and $D_{\mathrm{f}}$ are the DOS of an initial state and a final state, respectively, and $M_{\mathrm{fi}}$ is a transition matrix element. Since $D_{\mathrm{f}}$ is fixed in CFS, the curve roughly reflects $D_{\mathrm{i}}$ if the $h \nu$-dependence of $M_{\mathrm{fi}}$ is neglected. A kinetic energy of $0.2 \mathrm{eV}$ was selected as the fixed final state, as in a previous study. ${ }^{35}$

Figure 3 presents the CFS results for the different samples. Strong similarities in the shape and the magnitude of the low

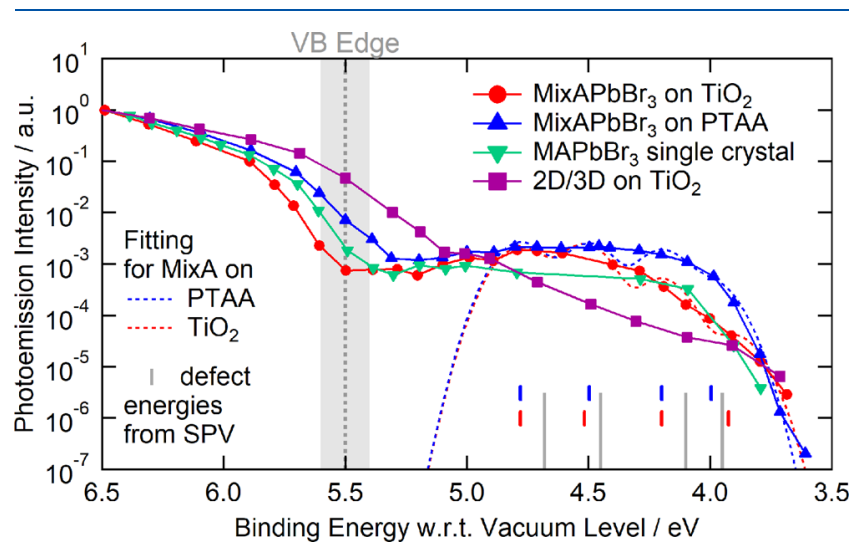

Figure 3. Comparison of the CFS of the different Br-based HaPs samples used in this study and curves fitted to $\mathrm{MixAPbBr}_{3}$ results (shown as dashed lines) based on defect energies obtained from SPV $(4.7,4.45,4.1$, and $3.95 \mathrm{eV}$ with respect to the vacuum level). The ordinate of every CFS curve was normalized by the intensity at the VB $\left(E_{\mathrm{B}}^{\mathrm{VL}}=6.5 \mathrm{eV}\right)$. The $x$-axis gives binding energies w.r.t. the $\mathrm{VL}$, i.e., $E_{\mathrm{B}}^{\mathrm{VL}}$. The notion of "VB Edge" refers to the 3D HaPs, as the VB Edge for the $2 \mathrm{D} / 3 \mathrm{D}$ is shifted to a lower binding energy.

binding energy feature are observed among the CFS spectra of the $\mathrm{MixAPbBr}_{3}$ films and the $\mathrm{MAPbBr}_{3}$ single crystal, suggesting that deep gap states are also present in the bulk of the $\mathrm{Br}-\mathrm{HaP}$ crystal at comparable densities since the sampling depth is on the order of $5 \mathrm{~nm}$, which we consider as bulk. Although many reports suggest that the deep gap states densities in single crystals are several orders of magnitude lower than those of thin films, ${ }^{31}$ it could be that our finding of rather similar deep gap state densities reflects differences due to the measurement method used, or suggests that the number of electrically active deep gap states is significantly smaller than the number of optically active deep gap states. By using the gap state energies previously determined for $\mathrm{MixAPbBr}_{3}$ films using modulated SPV ${ }^{4} E_{\mathrm{B}}^{\mathrm{VL}}=4.7,4.45,4.1$, and $3.95 \mathrm{eV}$, a relatively good fit was obtained to the CFS spectra, as shown in Figure 3. By comparing the CFS result of the $2 \mathrm{D} / 3 \mathrm{D} \mathrm{Br} \mathrm{HaP}$ (purple curve) to the $3 \mathrm{D}$-based perovskites spectra, it is evident that the deep gap state density is greatly reduced, explaining the high PLQE recently reported for the $2 \mathrm{D} / 3 \mathrm{D}$ films. ${ }^{20}$ The lower gap state density in the $2 \mathrm{D} / 3 \mathrm{D} \mathrm{HaP}$ could also be a result of an increased concentration of the $2 \mathrm{D}$ phase in the first $1-2 \mathrm{~nm}$ near surface; however, given the small electron kinetic energies involved, the sampling depth of HS-UPS, determined by the electron escape depth, will easily be up to $5 \mathrm{~nm}$, so that a significant part of the signal comes from the $3 \mathrm{D}$ phase, as also the bulk of the film is sampled.

Since the CBM lies at $E_{\mathrm{B}}^{\mathrm{VL}}=3.2 \mathrm{eV}$ and the defect bands extend from $\mathrm{VB}$ to $E_{\mathrm{B}}^{\mathrm{VL}} \sim 3.7 \mathrm{eV}-4.0 \mathrm{eV}$, the transition from occupied defect states to the $\mathrm{CB}$ starts to occur at a minimum $h \nu$ of $0.5 \mathrm{eV}-0.8 \mathrm{eV}$. This suggests that the region with $h \nu$ lower than where the Urbach tail located in the PDS in Figure 1a should contain signals arising from the defect-to-CB transition and that it is not just entirely background signal.

Next, we investigate the $h \nu$-dependence of the photoemission intensity to identify the character of the wave 

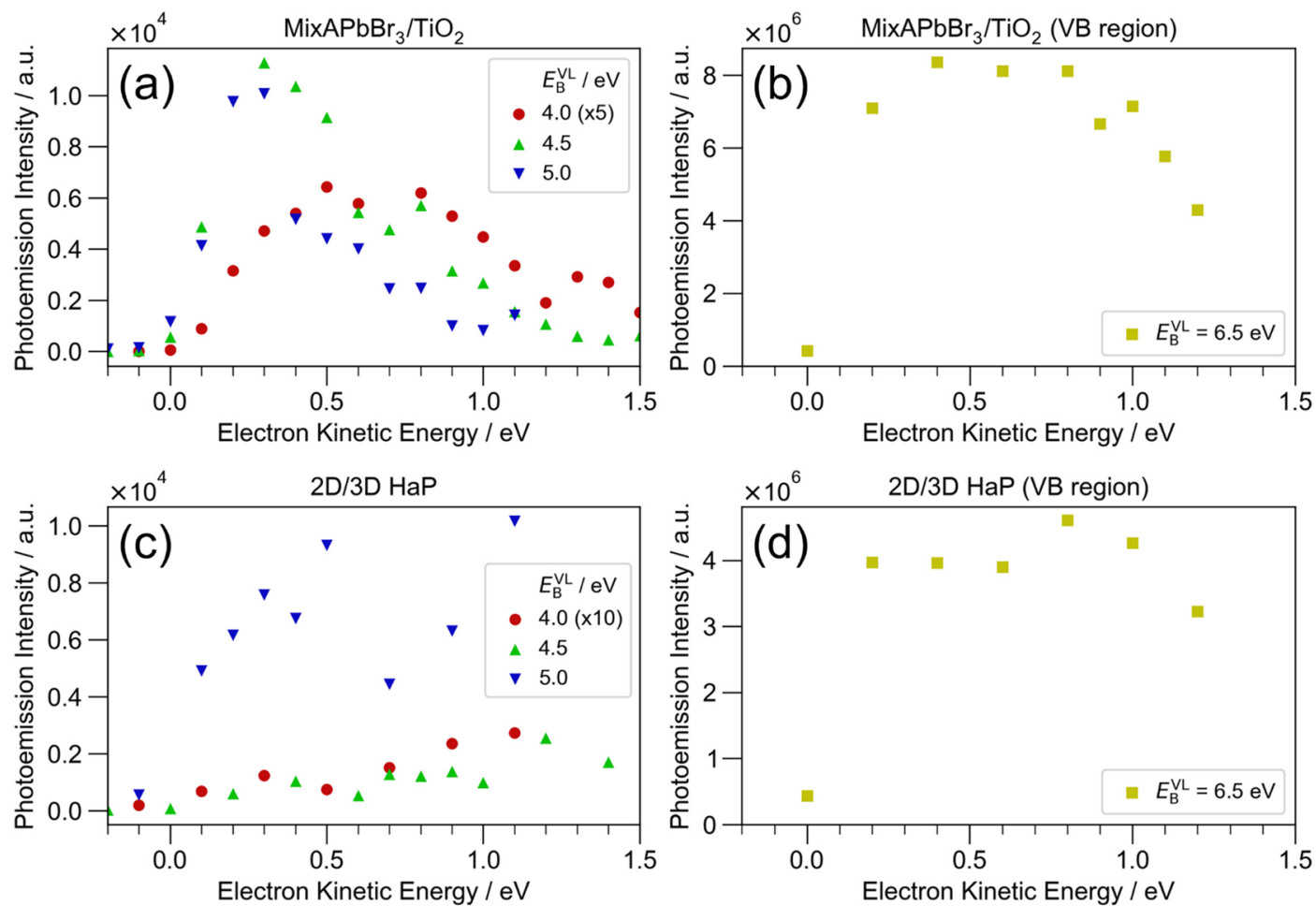

Figure 4. Intensity of photoelectrons emitted from energy levels of (a, c) 4.0, 4.5, and $5.0 \mathrm{eV}$ and (b, d) $6.5 \mathrm{eV}$ with respect to the vacuum level, plotted as a function of photoelectron kinetic energy (CIS plots). The symbol of each level corresponds to that indicating the energy position of the level in Figure 2.

functions. Focusing on the range around $E_{\mathrm{B}}^{\mathrm{VL}}=4.5 \mathrm{eV}$ in parts $\mathrm{a}$ and $\mathrm{b}$ of Figure 2, we see that the intensity at $h \nu \sim 4.8 \mathrm{eV}$ (blue spectrum) is larger than that at higher incident photon energy such as $h \nu \sim 5.5 \mathrm{eV}$ (cyan), while such a behavior is not apparent for the $2 \mathrm{D} / 3 \mathrm{D} \mathrm{HaP}$. To evaluate the dependence of the photoemission intensity on the photon energy, we employed a constant initial state (CIS) analysis, $^{36}$ in which the photoemission intensity from a specific initial state is plotted against the kinetic energy of photoelectrons $E_{\mathrm{K}}=h \nu-$ $E_{\mathrm{B}}^{\mathrm{VL}}$.

Figure 4a is a CIS plot for the $\mathrm{MixAPbBr}_{3}$ on $\mathrm{TiO}_{2}$, showing photoemission intensity from three different energy levels in the low binding energy feature. Their binding energies are $E_{\mathrm{B}}^{\mathrm{VL}}$ $=4.0,4.5$, and $5.0 \mathrm{eV}$, indicated by the arrows in Figure 2a. For all of the levels, the CIS curve has a peak within the range of $E_{\mathrm{K}}$ $=0.3-0.5 \mathrm{eV}$, revealing the prominent dependence of photoemission intensity on $E_{\mathrm{K}}$, and thus on $h \nu$. On the other hand, the CIS plot for the VB (Figure $4 \mathrm{~b}, E_{\mathrm{B}}^{\mathrm{VL}}=6.5 \mathrm{eV}$ ) does not show a pronounced change in intensity over the $E_{\mathrm{K}}=0.2-$ $1.0 \mathrm{eV}$ range.

Since every final state with kinetic energy $E_{\mathrm{K}}$ can be considered as a plane wave with a specific wavevector $k, M_{\mathrm{fi}}$ roughly corresponds to the Fourier transform of the initial state. Therefore, the CIS plot roughly reflects the $k$-space distribution of the initial state. If the plot shows a peak at $E_{\mathrm{K}}=$ $E_{\mathrm{K}}{ }^{\text {peak }}$, the wavenumber of the final state with $E_{\mathrm{K}}{ }^{\text {peak }}$ corresponds to the primary $k$-component of the initial state. In other words, wavelength matching between the initial and final states occurs at that wavenumber. The intensity in CIS includes $D_{\mathrm{f}}$ as well as $M_{\mathrm{fi}}$. Nevertheless, since the $D_{\mathrm{f}}$ depends only on $E_{\mathrm{K}}$ and is common to every $E_{\mathrm{B}}^{\mathrm{VL}}$, the distinction seen in CIS plots can be interpreted as differences in the wave function of the initial states. Thus, the trend in a CIS curve is a good tool to characterize initial state wave functions, and the distinct spectral shapes between parts $a$ and $b$ of Figures 4 are indicative of the disparity in the wave function between the VB and the low binding energy feature. In addition, the noticeable discrepancy in the peak position between the curves of $E_{\mathrm{B}}^{\mathrm{VL}}=$ $4.0 \mathrm{eV}$, which peaks at $E_{\mathrm{K}}=0.5 \mathrm{eV}$, and those of 4.5 and $5.0 \mathrm{eV}$ $\left(E_{\mathrm{K}}=0.3 \mathrm{eV}\right)$ suggests that the wave function differs even among the levels in the same low binding energy structure.

Concerning the $2 \mathrm{D} / 3 \mathrm{D} \mathrm{HaP}$, the shape of the CIS curve for the VB resembles that of $\mathrm{MixAPbBr}_{3}$ as seen in Figure $4 \mathrm{~d}$, which is expected, considering the common composition of those HaPs. Conversely, in contrast to the $\mathrm{MixAPbBr}_{3}$, the curve for the low binding energy feature in Figure $4 c$ hardly shows a peak-like structure, reflecting the absence of strong low binding energy features in the 2D/3D HS-UPS data (Figure 2c). Since the lack of defects was also confirmed by SPV, which used $h \nu=1-3 \mathrm{eV}$, it is not likely that the decrease of $M_{\mathrm{fi}}$ in the off-resonant condition leads to an underestimation of the DOS. These insights demonstrate that not only is HSUPS useful for elucidating the density of states over a much wider range than conventional UPS but also it allows differentiating between the character of the wave functions.

\section{CONCLUSIONS}

In conclusion, by using highly sensitive variable energy UPS, we were able to directly detect deep gap states in Br-based HaPs. PL measurements suggest that for the observed gap states, defect-to-band or band-to-defect transitions are mainly nonradiative or their densities are below the sensitivity of the used PL setup. We find that the deep gap states are also present in $\mathrm{MAPbBr}_{3}$ single crystals, and they need not necessarily be related (only) to the polycrystalline nature of the studied thin films. Incorporating a $2 \mathrm{D}$ precursor into the 
$\mathrm{HaP}$ composition greatly suppresses the number of deep gap states. Although the arrangement of the $2 \mathrm{D}$ and $3 \mathrm{D}$ components is not yet fully resolved, it is clear that $2 \mathrm{D} / 3 \mathrm{D}$ $\mathrm{HaP}$ mixtures are a promising route for increasing the efficiency of optoelectronic devices based on $\mathrm{Br}-\mathrm{HaPs}$. Our results provide a way to refine the question of what is "defect tolerance" in these family of materials. Since we find similar densities of optically active defects for both single crystals and thin films, that raises the issue of the possible presence defect densities that are optically active yet electrically inactive. Still, we note that the number of optically active defects is rather low since it could not be detected using PDS. Due to the comparably low density of defects present in HaPs and the very high dynamic range of the HS-UPS method, we suggest that HS-UPS is an essential method for directly probing defects in the HaP family of materials and testing for efficient passivation routes on the way to more efficient HaP-based devices in the future.

\section{ASSOCIATED CONTENT}

\section{(3) Supporting Information}

The Supporting Information is available free of charge at https://pubs.acs.org/doi/10.1021/acs.jpcc.0c11627.

HS-UPS measurement results of a $\mathrm{Cr} / \mathrm{Au}$ film, modulated SPV measurement results for the $\mathrm{MixAPbBr}_{3}$ compared to the $2 \mathrm{D} / 3 \mathrm{D}$ film, variable intensity $\mathrm{He}-1$ UPS measurements of the $\mathrm{MixAPbBr}_{3}$ film, and further details on the fitting of SPV results to the CFS plots presented in Figure 3 (PDF)

\section{AUTHOR INFORMATION}

\section{Corresponding Authors}

Igal Levine - Helmholtz-Zentrum Berlin für Materialien und Energie GmbH, 12489 Berlin, Germany; 이이.org/00000003-0406-2922; Email: igal.levine@helmholtz-berlin.de

Hisao Ishii - Center for Frontier Science, Chiba University, 263-8522 Chiba, Japan; Email: ishii130@faculty.chiba-u.jp

\section{Authors}

Kohei Shimizu - Center for Frontier Science, Chiba University, 263-8522 Chiba, Japan

Alberto Lomuscio - Laboratory for Photovoltaics, Université du Luxembourg, L-4422 Belvaux, Luxembourg;

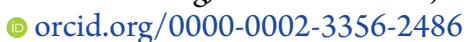

Michael Kulbak - Department of Materials \& Interfaces, Weizmann Institute of Science, Rehovot 76100, Israel

Carolin Rehermann - Helmholtz-Zentrum Berlin für Materialien und Energie GmbH, 12489 Berlin, Germany

Arava Zohar - Department of Materials \& Interfaces, Weizmann Institute of Science, Rehovot 76100, Israel; (1) orcid.org/0000-0002-8292-5968

Mojtaba Abdi-Jalebi - Institute for Materials Discovery, University College London, London WC1E 7JE, U.K.

Baodan Zhao - Cavendish Laboratory, University of Cambridge, Cambridge CB3 OHE, U.K.; State Key Laboratory of Modern Optical Instrumentation, College of Optical Science and Engineering, International Research Center for Advanced Photonics, Zhejiang University, Hangzhou 310027, P. R. China

Susanne Siebentritt - Laboratory for Photovoltaics, Université du Luxembourg, L-4422 Belvaux, Luxembourg
Fengshuo Zu - Institut für Physik \& IRIS Adlershof, Humboldt-Universität zu Berlin, 12489 Berlin, Germany

Norbert Koch - Helmholtz-Zentrum Berlin für Materialien und Energie GmbH, 12489 Berlin, Germany; Institut für Physik \& IRIS Adlershof, Humboldt-Universität zu Berlin, 12489 Berlin, Germany; 이이.org/0000-0002-60426447

Antoine Kahn - Department of Electrical Engineering, Princeton University, Princeton, New Jersey 08544, United States; orcid.org/0000-0002-1612-3350

Gary Hodes - Department of Materials \& Interfaces, Weizmann Institute of Science, Rehovot 76100, Israel; - orcid.org/0000-0001-7798-195X

Richard H. Friend - Cavendish Laboratory, University of Cambridge, Cambridge CB3 OHE, U.K.; (1) orcid.org/00000001-6565-6308

David Cahen - Department of Materials \& Interfaces, Weizmann Institute of Science, Rehovot 76100, Israel; BarIlan Institue for Nanotechnology \& Advanced Materials and Department of Chemistry, Bar-Ilan University, Ramat Gan 5290002, Israel; (1) orcid.org/0000-0001-8118-5446

Complete contact information is available at:

https://pubs.acs.org/10.1021/acs.jpcc.0c11627

\section{Author Contributions}

\&I.L. and K.S. contributed equally to this work

\section{Notes}

The authors declare no competing financial interest.

\section{ACKNOWLEDGMENTS}

I.L. thanks Davide Raffaele Ceratti for the preparation of $\mathrm{MAPbBr}_{3}$ single crystals, and Lars Korte and Dorothee Menzel for fruitful discussions. K.S and H.I would like to express their gratitude to the Japan Society for the Promotion of Science (JSPS) for funding via JSPS KAKENHI (Grants Nos. JP16H04222 and JP18J21921) as well as a JSPS Research Fellowship for Young Scientists (K.S.). M.A.-J. thanks Cambridge Materials Limited, Wolfson College, University of Cambridge, and the EPSRC (Grant No. EP/M005143/1) for their funding and technical support. In Luxembourg, this work was supported by Luxembourgish Fonds National de la Recherche (FNR). A.K. and D.C gratefully acknowledge support by the US-Israel Binational Science Foundation (BSF Grant No. 2018349). F.Z. and N.K. acknowledge funding by the DFG (Project Number 423749265 SPP2196). B.Z. acknowledges the National Natural Science Foundation of China (NSFC) (62005243), the National Natural Science Foundation of Zhejiang Province of China (LR21F050003), and the Fundamental Research Funds for the Central Universities (2020QNA5002). I.L acknowledges the Helmholtz Association for funding via the PEROSEED project. C.R. acknowledges financial support from the HI-SCORE research school of the Helmholtz Association and the HyPerCells Graduate School. D.C and A.K. thank Chiba University for hospitality as visiting professors. At the Weizmann Institute of Science (WIS), D.C. thanks the Yotam project, Ullmann Family Foundation, Dears Foundation, and WIS' Sustainability And Energy Research Initiative, SAERI, for support. 


\section{REFERENCES}

(1) Shockley, W.; Queisser, H. J. Detailed Balance Limit of Efficiency of P-n Junction Solar Cells. J. Appl. Phys. 1961, 32 (3), 510-519.

(2) Khadka, D. B.; Shirai, Y.; Yanagida, M.; Miyano, K. Degradation of Encapsulated Perovskite Solar Cells Driven by Deep Trap States and Interfacial Deterioration. J. Mater. Chem. C 2018, 6 (1), 162-170.

(3) Levine, I.; Gupta, S.; Brenner, T. M.; Azulay, D.; Millo, O.; Hodes, G.; Cahen, D.; Balberg, I. Mobility-Lifetime Products in MAPbI3 Films. J. Phys. Chem. Lett. 2016, 7 (24), 5219-5226.

(4) Levine, I.; Vera, O. G.; Kulbak, M.; Ceratti, D.-R.; Rehermann, C.; Márquez, J. A.; Levcenko, S.; Unold, T.; Hodes, G.; Balberg, I.; et al. Deep Defect States in Wide-Band-Gap ABX 3 Halide Perovskites. ACS Energy Lett. 2019, 4 (5), 1150-1157.

(5) Baumann, A.; Väth, S.; Rieder, P.; Heiber, M. C.; Tvingstedt, K.; Dyakonov, V. Identification of Trap States in Perovskite Solar Cells. J. Phys. Chem. Lett. 2015, 6, 2350.

(6) Zhang, F.; Hamill, J. C.; Loo, Y.; Kahn, A. Gap States in Methylammonium Lead Halides: The Link to Dimethylsulfoxide? Adv. Mater. 2020, 32 (42), 2003482.

(7) Wu, X.; Trinh, M. T.; Niesner, D.; Zhu, H.; Norman, Z.; Owen, J. S.; Yaffe, O.; Kudisch, B. J.; Zhu, X. Y. Trap States in Lead Iodide Perovskites. J. Am. Chem. Soc. 2015, 137 (5), 2089-2096.

(8) Sueyoshi, T.; Fukagawa, H.; Ono, M.; Kera, S.; Ueno, N. LowDensity Band-Gap States in Pentacene Thin Films Probed with Ultrahigh-Sensitivity Ultraviolet Photoelectron Spectroscopy. Appl. Phys. Lett. 2009, 95 (18), 183303.

(9) Machida, S.; Ozawa, Y.; Takahashi, J.; Tokairin, H.; Nakayama, Y.; Ishii, H. Low-Energy Photoemission Study of $\mathrm{C}_{60} /$ Rubrene/Au Interfaces in Practical Device Thickness. Appl. Phys. Express 2013, 6 (2), 025801.

(10) Schulz, P.; Edri, E.; Kirmayer, S.; Hodes, G.; Cahen, D.; Kahn, A. Interface Energetics in Organo-Metal Halide Perovskite-Based Photovoltaic Cells. Energy Environ. Sci. 2014, 7 (4), 1377.

(11) Schulz, P.; Tiepelt, J. O.; Christians, J. A.; Levine, I.; Edri, E.; Sanehira, E. M.; Hodes, G.; Cahen, D.; Kahn, A. High-Work-Function Molybdenum Oxide Hole Extraction Contacts in Hybrid OrganicInorganic Perovskite Solar Cells. ACS Appl. Mater. Interfaces 2016, 8 (46), 31491

(12) Endres, J.; Egger, D. A.; Kulbak, M.; Kerner, R. A.; Zhao, L.; Silver, S. H.; Hodes, G.; Rand, B. P.; Cahen, D.; Kronik, L.; et al. Valence and Conduction Band Densities of States of Metal Halide Perovskites: A Combined Experimental-Theoretical Study. J. Phys. Chem. Lett. 2016, 7 (14), 2722-2729.

(13) Boehm, A. M.; Wieser, J.; Butrouna, K.; Graham, K. R. A New Photon Source for Ultraviolet Photoelectron Spectroscopy of Organic and Other Damage-Prone Materials. Org. Electron. 2017, 41, 9-16.

(14) Sato, T.; Kinjo, H.; Yamazaki, J.; Ishii, H. $10^{15} \mathrm{Cm}^{-3} \mathrm{EV}^{-1}$ Level Detection of Density of States of a p-Type Polymer by $h \nu$ Dependent High-Sensitivity Ultraviolet Photoemission Spectroscopy. Appl. Phys. Express 2017, 10 (1), 011602.

(15) Yamaguchi, Y.; Shimizu, K.; Matsuzaki, A.; Sano, D.; Sato, T.; Tanaka, Y.; Ishii, H. Gap States of a Polyethylene Model Oligomer Observed by Using High-Sensitivity Ultraviolet Photoelectron Spectroscopy. IEICE Trans. Electron. 2019, E102.C (2), 168-171.

(16) Puschnig, P.; Berkebile, S.; Fleming, A. J.; Koller, G.; Emtsev, K.; Seyller, T.; Riley, J. D.; Ambrosch-Draxl, C.; Netzer, F. P.; Ramsey, M. G. Reconstruction of Molecular Orbital Densities from Photoemission Data. Science (Washington, DC, U. S.) 2009, 326 (5953), 702-706

(17) Musiienko, A.; Moravec, P.; Grill, R.; Praus, P.; Vasylchenko, I.; Pekarek, J.; Tisdale, J.; Ridzonova, K.; Belas, E.; Landová, L.; et al. Deep Levels, Charge Transport and Mixed Conductivity in Organometallic Halide Perovskites. Energy Environ. Sci. 2019, 12 (4), 14131425.

(18) Bouduban, M. E. F.; Queloz, V. I. E.; Caselli, V. M.; Cho, K. T.; Kirmani, A. R.; Paek, S.; Roldan-Carmona, C.; Richter, L. J.; Moser, J. E.; Savenije, T. J.; et al. Crystal Orientation Drives the Interface
Physics at Two/Three-Dimensional Hybrid Perovskites. J. Phys. Chem. Lett. 2019, 10, 5713.

(19) Cheng, L.-P.; Huang, J.-S.; Shen, Y.; Li, G.-P.; Liu, X.-K.; Li, W.; Wang, Y.-H.; Li, Y.-Q.; Jiang, Y.; Gao, F.; et al. Efficient CsPbBr ${ }_{3}$ Perovskite Light-Emitting Diodes Enabled by Synergetic Morphology Control. Adv. Opt. Mater. 2019, 7 (4), 1801534.

(20) Zhao, B.; Lian, Y.; Cui, L.; Divitini, G.; Kusch, G.; Ruggeri, E.; Auras, F.; Li, W.; Yang, D.; Zhu, B.; et al. Efficient Light-Emitting Diodes from Mixed-Dimensional Perovskites on a Fluoride Interface. Nat. Electron. 2020, 3, 704.

(21) Azulay, D.; Levine, I.; Gupta, S.; Barak-Kulbak, E.; Bera, A.; San, G.; Simha, S.; Cahen, D.; Millo, O.; Hodes, G.; et al. On the Influence of Multiple Cations on the In-Gap States and Phototransport Properties of Iodide-Based Halide Perovskites. Phys. Chem. Chem. Phys. 2018, 20, 24444.

(22) Sato, T.; Kinjo, H.; Yamazaki, J.; Ishii, H. $10^{15} \mathrm{Cm}^{-3} \mathrm{EV}^{-1}$ Level Detection of Density of States of a p-Type Polymer by $h \nu$ Dependent High-Sensitivity Ultraviolet Photoemission Spectroscopy. Appl. Phys. Express 2017, 10 (1), 011602.

(23) Ceratti, D. R.; Rakita, Y.; Cremonesi, L.; Tenne, R.; Kalchenko, V.; Elbaum, M.; Oron, D.; Potenza, M. A. C.; Hodes, G.; Cahen, D. Self-Healing Inside $\mathrm{APbBr}_{3}$ Halide Perovskite Crystals. Adv. Mater. 2018, 30 (10), 1706273.

(24) De Wolf, S.; Holovsky, J.; Moon, S.-J.; Löper, P.; Niesen, B.; Ledinsky, M.; Haug, F.-J.; Yum, J.-H.; Ballif, C. Organometallic Halide Perovskites: Sharp Optical Absorption Edge and Its Relation to Photovoltaic Performance. J. Phys. Chem. Lett. 2014, 5 (6), 10351039.

(25) Zohar, A.; Kulbak, M.; Levine, I.; Hodes, G.; Kahn, A.; Cahen, D. What Limits the Open-Circuit Voltage of Bromide PerovskiteBased Solar Cells? ACS Energy Lett. 2019, 4, 1-7.

(26) Rappich, J.; Lang, F.; Brus, V. V.; Shargaieva, O.; Dittrich, T.; Nickel, N. H. Light-Induced Defect Generation in $\mathrm{CH}_{3} \mathrm{NH}_{3} \mathrm{PbI}_{3}$ Thin Films and Single Crystals. Sol. RRL 2020, 4 (2), 1900216.

(27) Chen, C.; Hu, X.; Lu, W.; Chang, S.; Shi, L.; Li, L.; Zhong, H.; Han, J.-B. Elucidating the Phase Transitions and TemperatureDependent Photoluminescence of $\mathrm{MAPbBr}_{3}$ Single Crystal. J. Phys. D: Appl. Phys. 2018, 51 (4), 045105.

(28) Rey, G.; Spindler, C.; Babbe, F.; Rachad, W.; Siebentritt, S.; Nuys, M.; Carius, R.; Li, S.; Platzer-Björkman, C. Absorption Coefficient of a Semiconductor Thin Film from Photoluminescence. Phys. Rev. Appl. 2018, 9 (6), 064008.

(29) Ledinsky, M.; Schönfeldová, T.; Holovský, J.; Aydin, E.; Hájková, Z.; Landová, L.; Neyková, N.; Fejfar, A.; De Wolf, S. Temperature Dependence of the Urbach Energy in Lead Iodide Perovskites. J. Phys. Chem. Lett. 2019, 10 (6), 1368-1373.

(30) Liu, Y.; Yang, Z.; Cui, D.; Ren, X.; Sun, J.; Liu, X.; Zhang, J.; Wei, Q.; Fan, H.; Yu, F.; et al. Two-Inch-Sized Perovskite $\mathrm{CH} 3 \mathrm{NH} 3 \mathrm{PbX} 3(\mathrm{X}=\mathrm{Cl}, \mathrm{Br}, \mathrm{I})$ Crystals: Growth and Characterization. Adv. Mater. 2015, 27 (35), 5176-5183.

(31) Kumar, S.; Hodes, G.; Cahen, D. Defects in Halide Perovskites: The Lattice as a Boojum? MRS Bull. 2020, 45 (6), 478-484.

(32) Zu, F.; Wolff, C. M.; Ralaiarisoa, M.; Amsalem, P.; Neher, D.; Koch, N. Unraveling the Electronic Properties of Lead Halide Perovskites with Surface Photovoltage in Photoemission Studies. ACS Appl. Mater. Interfaces 2019, 11 (24), 21578-21583.

(33) Korte, L.; Schmidt, M. Investigation of Gap States in Phosphorous-Doped Ultra-Thin a-Si:H by near-UV Photoelectron Spectroscopy. J. Non-Cryst. Solids 2008, 354 (19-25), 2138-2143.

(34) Johnson, B.; Korte, L.; Lußky, T.; Klaer, J.; Lauermann, I. CuInS2 -CdS Heterojunction Valence Band Offset Measured with near-UV Constant Final State Yield Spectroscopy. J. Appl. Phys. 2009, 106 (7), 073712.

(35) Sebastiani, M.; Di Gaspare, L.; Capellini, G.; Bittencourt, C.; Evangelisti, F. Low-Energy Yield Spectroscopy as a Novel Technique for Determining Band Offsets: Application to the c-Si(100)/a-Si:H Heterostructure. Phys. Rev. Lett. 1995, 75 (18), 3352-3355. 
(36) Ley, L.; Cardona, M.; Pollak, R. A. Photoemission in Semiconductors; Springer: Berlin and Heidelberg, Germany, 1979; pp 11-172. 